\title{
Attractiveness of a Four-component Pheromone Blend to Male Navel Orangeworm Moths
}

\author{
Hiroo Kanno • L. P. S. Kuenen • Kimberly A. Klingler • \\ Jocelyn G. Millar • Ring T. Cardé
}

Received: 11 January 2010 /Revised: 22 April 2010 /Accepted: 29 April 2010 /Published online: 16 May 2010

(C) The Author(s) 2010. This article is published with open access at Springerlink.com

\begin{abstract}
The attractiveness to male navel orangeworm moth, Amyelois transitella, of various combinations of a four-component pheromone blend was measured in windtunnel bioassays. Upwind flight along the pheromone plume and landing on the odor source required the simultaneous presence of two components, $(11 Z, 13 Z)$ hexadecadienal and $(3 Z, 6 Z, 9 Z, 12 Z, 15 Z)$-tricosapentaene, and the addition of either $(11 Z, 13 Z)$-hexadecadien-1-ol or $(11 Z, 13 E)$-hexadecadien-1-ol. A mixture of all four components produced the highest levels of rapid source location and source contact. In wind-tunnel assays, males did not seem to distinguish among a wide range of ratios of any of the three components added to $(11 Z, 13 Z)$-hexadecadienal. Dosages of 10 and $100 \mathrm{ng}$ of the 4-component blend produced higher levels of source location than dosages of 1 and $1,000 \mathrm{ng}$.
\end{abstract}

Key Words Lepidoptera $\cdot$ Pyralidae $\cdot(11 Z, 13 Z)$ hexadecadienal $\cdot(3 Z, 6 Z, 9 Z, 12 Z, 15 Z)$-tricosapentaene . (11Z,13Z)-hexadecadien-1-ol $\cdot(11 Z, 13 E)$-hexadecadien-1-ol

Electronic supplementary material The online version of this article (doi:10.1007/s10886-010-9799-x) contains supplementary material, which is available to authorized users.

H. Kanno $\cdot$ K. A. Klingler · J. G. Millar $\cdot$ R. T. Cardé $(\bowtie)$

Department of Entomology, University of California,

Riverside, CA 92521, USA

e-mail: ring.carde@ucr.edu

L. P. S. Kuenen

USDA-Agricultural Research Service,

9611 South Riverbend Avenue,

Parlier, CA 93648, USA

\section{Introduction}

The navel orangeworm, Amyelois transitella (Walker) (Lepidoptera: Pyralidae), is a major pest of the multibillion dollar almond, pistachio, and walnut crops in California. The sex pheromone produced by the female navel orangeworm moth was characterized originally as a single component, (11Z,13Z)-hexadecadienal (Z11,Z13-16:Ald) (Coffelt et al. 1979a). Although this compound stimulated male activation, wing fanning, and upwind movement in laboratory bioassays (Coffelt et al. 1979b), its activity as an attractant for field traps was inconsistent, and it was only weakly attractive when compared to virgin female moths (e.g., Kuenen et al. 2001, 2010). In recent studies, a number of additional compounds have been identified from pheromone gland extracts (Leal et al. 2005; Kuenen et al. 2010), including analogs of Z11,Z13-16:Ald, such as $(11 Z, 13 E)$ hexadecadienal (Z11,E13-16:Ald), (11E,13Z)-hexadecadienal (E11,Z13-16:Ald), (11Z,13Z)-hexadecadien-1-ol (Z11,Z1316:OH), (11Z,13Z)-hexadecadien-1-yl acetate (Z11,Z13-16: Ac), hexadecanal (16:Ald), (Z)-11-hexadecenal (Z11-16: Ald), (Z)-13-hexadecenal (Z13-16:Ald), ethyl hexadecanoate (16:COOEt, ethyl palmitate), and ethyl $(11 Z, 13 Z)$-hexadecadienoate (Z11,Z13-16:COOEt). Two unusual polyunsaturated hydrocarbons, $(3 Z, 6 Z, 9 Z, 12 Z, 15 Z)$-tricosapentaene $\left(C_{23}\right.$ pentaene) and $(3 Z, 6 Z, 9 Z, 12 Z, 15 Z)$-pentacosapentaene $\left(C_{25}\right.$ pentaene), also were found in gland extracts. The first group of compounds is typical of the so-called Type I lepidopteran pheromones, that is, compounds with $\mathrm{C}_{10}$ to $\mathrm{C}_{18}$ straight chains terminating in alcohol, aldehyde, or ester functions. In contrast, the two pentaenes belong to the Type II lepidopteran pheromones, characterized by polyunsaturated hydrocarbons and related epoxides and ketones, with $\mathrm{C}_{17}$ to $\sim \mathrm{C}_{25}$ carbon chain lengths (Ando et al. 2004). Thus, this insect is one of the first examples of a 
moth species that might use a combination of both Type I and Type II compounds in its pheromone blend. In field trials, Leal et al. (2005) demonstrated that blends of five of these components, in combination with Z11,Z13-16:Ald, were attractive to male moths, but they did not report further attempts to refine the blend. Thus, it remained unclear as to which specific compounds were necessary and sufficient to constitute an attractive blend. More rigorous attempts to delineate the true blend by using a combination of wind tunnel and field bioassays spanning several field seasons, provided evidence that the pheromone blend is composed of at least four components, Z11,Z13-16:Ald, Z11,Z13-16: $\mathrm{OH},(11 Z, 13 E)$-hexadecadien-1-ol (Z11,E13-16:OH), and $\mathrm{C}_{23}$ pentaene (Kuenen et al. 2010). Furthermore, Kuenen et al. (2010) found no evidence that other components found in gland extracts, Z11,E13-16:Ald, E11,Z13-16: Ald, Z11,Z13-16:Ac, Z11-16:Ald, 16:Ald, hexadecan-1$\mathrm{ol}$, and $\mathrm{C}_{25}$ pentaene, were components of the pheromone blend.

The aim of the work reported here was to delineate and define the optimal attractive pheromone blend of the navel orangeworm. Our specific objectives were to conduct wind tunnel bioassays to assess: 1 . Attraction of male moths to the major component, Z11,Z13-16:Ald, plus one, two, or three of the additional minor components that had been implicated in previous studies; 2. attraction to various blends in which the proportion of a single component was varied systematically while holding the ratio of the other components fixed; 3 . the effect of adding other compounds, identified in pheromone gland extracts, to an optimized blend of 4 components; 4 . the effect of dose of the pheromone blend on responses of male $A$. transitella.

\section{Methods and Materials}

Insects Our colony of $A$. transitella was established with eggs obtained from the USDA, Agricultural Research Service, Parlier, CA, USA, from a colony founded from individuals collected in 2001 from walnuts in Fresno County, CA, USA. Larvae were reared in 3.8-1 jars on a red flaky wheat diet (Coffelt et al. 1979a). Cultures were maintained at $26^{\circ} \mathrm{C}$, with a photoperiod of $16: 8 \mathrm{~L}: \mathrm{D}$ and 50 $60 \%$ relative humidity. Approximately 80 last instar males were removed daily from the colony and placed in $180-\mathrm{ml}$ plastic cups (20 per cup). These larvae were held in a controlled temperature cabinet separated from the rearing colony, under a reversed photoperiod, 14:10L:D regime at $25^{\circ} \mathrm{C}$. Males that emerged each day were transferred to a $30 \times 30 \times 30 \mathrm{~cm}$ plastic screen cage, where they were held with access to $10 \%$ sucrose solution. All individuals used in bioassays were $2-4$-d-old.
Wind-tunnel Bioassays A wind tunnel $(1 \mathrm{~m}$ wide $\times 1 \mathrm{~m}$ high $\times 3 \mathrm{~m}$ long), described in Justus et al. (2002), was used for all experiments. Because male A. transitella often ascend $15 \mathrm{~cm}$ or more after take-off in a pheromone plume, the $1 \mathrm{~m}$ height of this tunnel was essential for the bioassay. The wind speed was set to $50-60 \mathrm{~cm} \mathrm{~s}^{-1}$ by regulating two variable-speed fans, one on the tunnel input and the other on the exhaust. The wind speed was verified with a Barnant Tri-Sense (Cole-Parmer 37000-61) anemometer. All air flowing through the tunnel was exhausted from the building. The tunnel's floor was covered with white fabric, and red paper circles, $5.4 \mathrm{~cm}$ diam., set randomly $\left(30\right.$ per $\left.\mathrm{m}^{2}\right)$ on the floor in a non-overlapping pattern to provide nondirectional visual cues. All experiments were conducted at $24-26^{\circ} \mathrm{C}$ with a relative humidity of $50-60 \%$. Two lateral banks of $25-\mathrm{W}$, clear red (Philips Colortone R) and 25-W tungsten lights, on each side of the room, illuminated the ceiling and were set with a rheostat to provide a diffuse light of 7-10 lux in the wind tunnel. A Sanyo VCB-3512T monochrome CCD camera (shutter speed, $1 / 100 \mathrm{sec}$ ), with a $75 \mathrm{~mm}$ lens, was mounted at the side of the wind tunnel to record male behavior at the pheromone source (see below).

All experiments were conducted during the last $3 \mathrm{hr}$ of scotophase, the period when female $A$. transitella call and mate (Coffelt et al. 1979b). Test males were brought into the assay room and transferred individually into a small wire mesh cylindrical $(3 \mathrm{~cm}$ diam. $\times 5 \mathrm{~cm}$ high) cage, with one end open and the other closed. Males were acclimated to the conditions of the assay room for $60-180 \mathrm{~min}$. before being tested. To test male responsiveness to a pheromone/ odor source, a cage was placed, closed-end facing upwind, on a wire-mesh stand set $21 \mathrm{~cm}$ above the tunnel floor and $160 \mathrm{~cm}$ downwind of the pheromone source. Once a male began wing fanning, the cage was rotated $180^{\circ}$, thereby allowing him to initiate upwind flight. Test males were used once and discarded.

The following were noted:

Percentage taking flight: proportion of males initiating wing fanning that take flight within $1 \mathrm{~min}$. of the cage being placed on the stand in the pheromone plume.

Latency of taking flight: time from cage being placed on stand in pheromone plume until the initiation of flight.

Percentage of source contact: proportion of males locking onto the plume that landed on the pheromone source.

Latency of source contact: time from initiation of flight to landing on the pheromone source.

Duration of source contact: time of continuous contact with the source after landing. All males walked and wing fanned while in contact with the source. If a male 
flew off the source and then re-contacted it (a relatively rare occurrence), we did not consider time spent in a subsequent landing in our measurement of duration of source contact. Observations were terminated after 2 min. of continuous source contact.

Odor Sources All compounds used were synthesized as described in detail in Kuenen et al. (2010). Stock solutions ( $1 \mathrm{mg} / \mathrm{ml}$ in heptane) were formulated from compounds that were re-purified (see Kuenen et al. 2010) immediately before making up the solutions, and had the following purities (known impurities): Z11,Z13-16:Ald, $>98 \%$ pure ( 0.6\% Z11,E13-16:Ald and $\sim 0.6 \%$ E11,Z13-16:Ald); Z11, $\mathrm{Z13}-16: \mathrm{OH},>98 \%$ chemically and isomerically pure; $\mathrm{C}_{23}$ pentaene, $>99 \%$ pure; Z11,E13-16:OH, $>96 \%$ pure. Working solutions were prepared by dilution of stock solutions in hexane. Point sources of odor were created by applying $20 \mu \mathrm{l}$ of a hexane solution of a particular blend to a $1-\mathrm{cm}$ diam. filter paper disk (Whatman \#1) mounted on a \#3 insect pin. The solvent was allowed to evaporate for $3 \mathrm{~min}$. before the paper disks were placed in glass vials until used (within $2 \mathrm{hr}$ ). The filter paper disk was pinned vertically into the eraser of a pencil, $22 \mathrm{~cm}$ above the tunnel floor. Each source was placed $100 \mathrm{~cm}$ from the upwind end of the tunnel and used for no longer than $20 \mathrm{~min}$.

Statistical Analyses In each experiment, a treatment was blocked daily as a group of five males; within the block, five males were tested consecutively. The order in which treatments were tested was randomized. All treatments were tested on each test day. The proportions of individuals initiating flight or contacting the lure were analyzed for differences by treatment using a $G$-test in SAS 9.1 (SAS Institute 2004). Results from asymptotic and exact tests were the same in all instances. Results of the exact test were reported because effective sample sizes for moths making contact with the lure in some instances were small. Time to initiation of flight, time to lure contact, and the duration of contact, were compared by treatment using Minitab's Regression with Life Data in Minitab 14 (Minitab Statistical Software 2004). The data for each of the above variables were determined to fit a lognormal distribution adequately $(P>0.94)$. Treatments with $N<10$ were excluded from analyses, but their means and standard deviations are reported.

\section{Results}

Our first experiment tested the effects of addition of one, two, or all three, of these compounds added to Z11,Z13-16:
Ald (Fig. 1) in the 100:100:5:15 ratio used by Kuenen et al. (2010). Z11,Z13-16:Ald, as a single component, elicited some activation and flight initiation (59\%), but no oriented upwind flight along the plume; no moths reached the

\section{a}

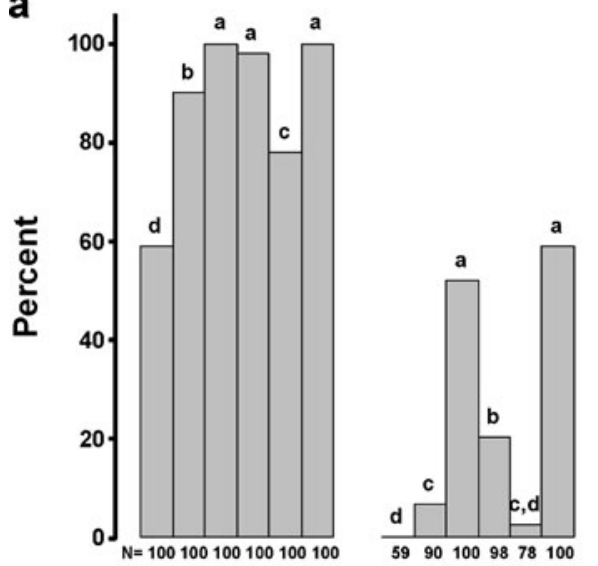

b

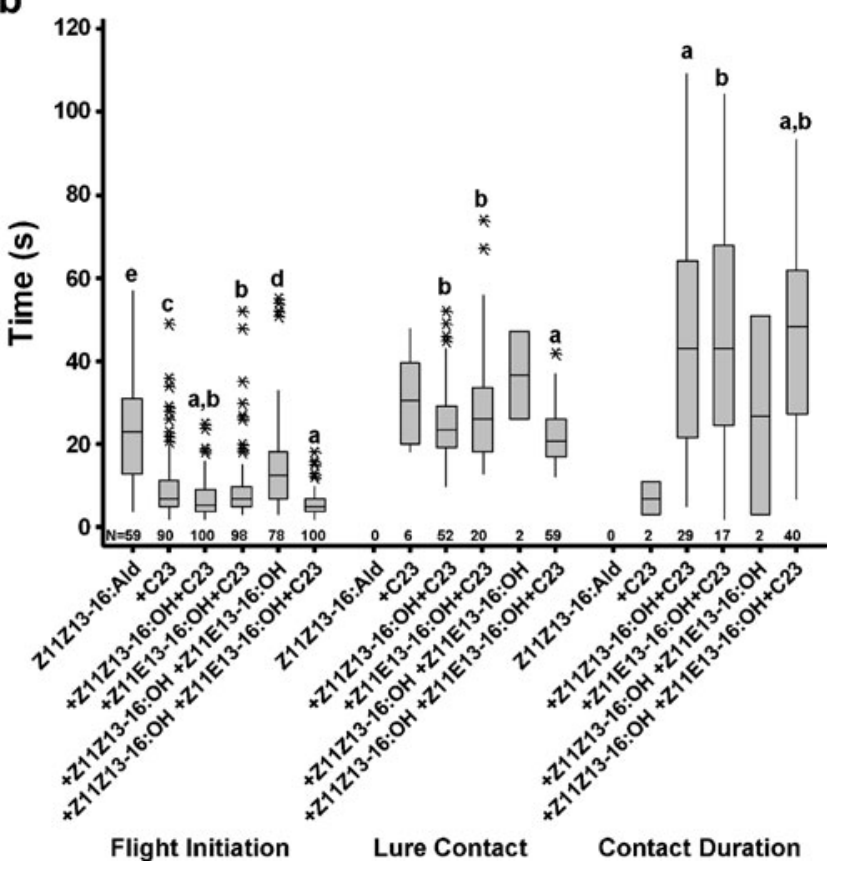

Fig. 1 Flight responses of male Amyelois transitella to various pheromone blends containing (11Z,13Z)-hexadecadienal (Z11Z13-16: Ald; in all blends), (3Z,6Z,9Z,12Z,15Z)-tricosapentaene (C23), (11Z,13Z)hexadecadien-1-ol (Z11Z13-16:OH), or (11Z,13E)-hexadecadien-1-ol (Z11E13-16:OH). a Percentage of males that initiated flight within $60 \mathrm{sec}$ of exposure to lure, and percentage of males that contacted a lure. $\mathrm{N}$ indicates the number of males tested for each response. $\mathbf{b}$ Time to flight initiation (censored at $60 \mathrm{sec}$ ), time to lure contact, and contact duration (censored at $120 \mathrm{sec}$ ). Boxes indicate 25 th and 75 th percentiles, bars inside boxes are medians, whiskers denote the range, and asterisks represent outliers. Z11Z13-16:Ald, Z11Z13-16:Ald + C23 and Z11Z1316:Ald + Z11Z13-16:OH + Z11E13-16:OH were not included in the analysis of time to contact and contact duration because of inadequate sample sizes. $\mathrm{N}$ indicates the number of males tested for each response. Different letters above columns or boxes [in (a) or (b)] indicate significant $(P<0.05)$ differences 
source. Addition of $\mathrm{C}_{23}$ pentaene to Z11,Z13-16:Ald increased the proportion of moths initiating flight, and reduced the mean latency to flight, but the percentage of moths contacting the source was still low (6.7\%). Similarly, the blend of Z11,Z13-16:Ald + Z11,Z13-16:OH + Z11,E13$16: \mathrm{OH}$ resulted in greater flight initiation and a shorter mean latency to flight than recorded to Z11,Z13-16:Ald alone but, again, there were few source contacts $(2.6 \%)$. Addition of either Z11,Z13-16:OH or Z11,E13-16:OH, or both, to the blend of Z11,Z13-16:Ald and $C_{23}$ pentaene, resulted in high levels of flight initiation (98-100\%), and short mean latencies $(7.3-9.6 \mathrm{sec})$.

The 4-component mixture evoked the most rapid initiation of flight, followed by the two 3-component mixtures containing $\mathrm{C}_{23}$ pentaene, the mixture containing Z11,Z13-16:Ald and $C_{23}$ pentaene, and the remainder of the treatments (Fig. 1). The time of source location followed the same trends seen in percentages of source location. There were 3 treatments that had sufficient numbers of males locating the source that permitted an analysis of latencies of source location (Fig. 1). The 4-component mixture evoked the most rapid source location, followed by the two 3-component mixtures containing $\mathrm{C}_{23}$ pentaene. The duration of source contact was longest for the 4component blend and the Z11,Z13-16:Ald, Z11,Z13-16: $\mathrm{OH}$, and $\mathrm{C}_{23}$ pentaene blend, and somewhat shorter for the mixture of Z11,Z13-16:Ald, Z11,E13-16:OH, and $\mathrm{C}_{23}$ pentaene.

Taken together, these comparisons demonstrate that a high proportion of source location required Z11,Z13-16: Ald, $\mathrm{C}_{23}$ pentaene, and either Z11,Z13-16:OH or Z11,E13$16: \mathrm{OH}$, or both. Although the 3-component blend of Z11, Z13-16:Ald, Z11,Z13-16:OH, and $\mathrm{C}_{23}$ pentaene was statistically indistinguishable from the full 4-component blend for percentage of moths locating the pheromone source, the 4-component blend evoked significantly more rapid flight to the source, supporting the role of Z11,E13-16:OH as a component of the optimal blend. In a follow-up wind tunnel test, aimed at verifying the role of Z11,E13-16:OH and comparing the same series of blend components, the proportion of moths contacting the lure was highest (all 3 treatments differed at $P<0.05$, Hantel-Haenszel-Cochran test) for the 4-component mixture (80.0\%), over the $Z 11$, Z13-16:Ald, Z11,Z13-16:OH and $\mathrm{C}_{23}$ pentaene blend (56.7\%), and the Z11,Z13-16:Ald, Z11,E13-16:OH and C23 pentaene mixture $(21.7 \%)$, confirming that all 4 compounds are involved in eliciting the full behavioral response.

The second experiment tested the effects of adding different proportions of $\mathrm{C}_{23}$ pentaene to a base blend, consisting of Z11,Z13-16:Ald, Z11,Z13-16:OH, and Z11, E13-16:OH (100:100:5). Although the $C_{23}$ pentaene was required for source location, males were remarkably insensitive to the percentage (from 1.5 to $150 \%$ of $\mathrm{Z} 11$, Z13-16:Ald) of this compound in the 4-component blend (Fig. 2). Based on these results, in further tests we used a 100:15 ratio of Z11,Z13-16:Ald to $C_{23}$ pentaene, equivalent to the optimum ratio used in bioassays of Kuenen et al. (2010).

In the third experiment, the proportion of Z11,Z13-16: $\mathrm{OH}$ was varied while holding the ratio of Z11,Z13-16:Ald, $\mathrm{C}_{23}$ pentaene, and Z11,E13-16:OH constant (100:15:5). Four-component blends, that varied greatly in the proportion of Z11,Z13-16:OH, elicited similar levels of attraction

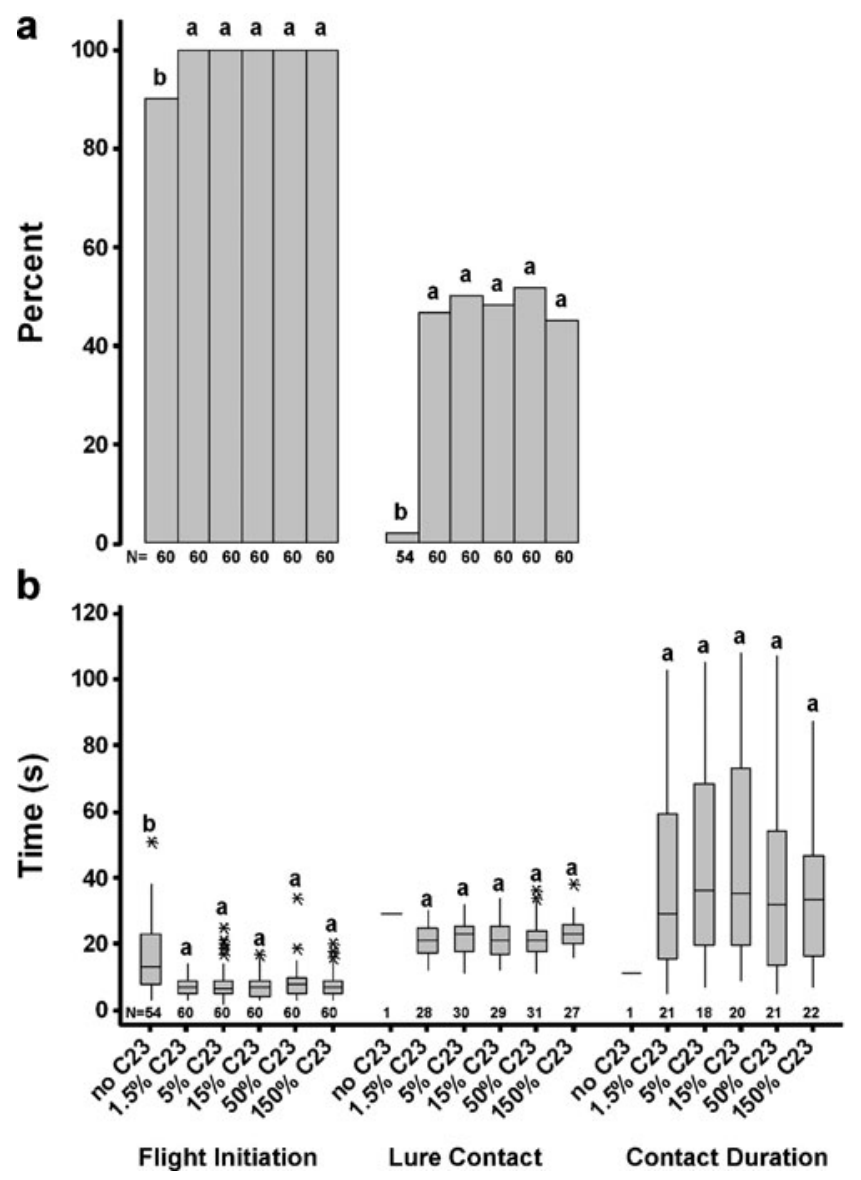

Fig. 2 Flight responses of male Amyelois transitella to pheromone blends with different amounts of $(3 Z, 6 Z, 9 Z, 12 Z, 15 Z)$-tricosapentaene (C23). All treatments contain (11Z,13Z)-hexadecadienal (10 ng), (11Z,13Z)-hexadecadien-1-ol, and $(11 Z, 13 E)$-hexadecadien-1-ol in a $100: 100: 5$ ratio. The percentage of $C 23$ is relative to the amount of $(11 Z, 13 Z)$-hexadecadienal. a Percentage of males that initiated flight within $60 \mathrm{sec}$ of exposure to lure, and percentage of males that contacted a lure. $\mathrm{N}$ indicates the number of males tested for each response. b Time to flight initiation (censored at $60 \mathrm{sec}$ ), time to lure contact, and contact duration (censored at $120 \mathrm{sec}$ ). Boxes indicate 25th and 75th percentiles, bars inside boxes are medians, whiskers denote the range, and asterisks represent outliers. The treatment lacking C23 was not included in the analysis of time to contact and contact duration due to inadequate sample size. $\mathrm{N}$ indicates the number of males exhibiting a response. Different letters above columns or boxes [in (a) or (b)] indicate significant $(P<0.05)$ differences 
and source contact by males; ratios of $\mathrm{Z} 11, \mathrm{Z} 13-16: \mathrm{OH}$ to Z11,Z13-16:Ald of 30:100 to 300:100 were statistically indistinguishable for all metrics (Fig. 3). Thus, for subsequent experiments, we used the intermediate ratio of 100:100 Z11,Z13-16:Ald to Z11,Z13-16:OH.

Varying the proportion of Z11,E13-16:OH, while holding the ratio of the three other components constant (Z11,Z13-16: Ald to Z11,Z13-16:OH to $\mathrm{C}_{23}$ pentaene, 100:100:15), also had little effect on male responses (Fig. 4). Percent source contact increased with inclusion of Z11,E13-16:OH in the
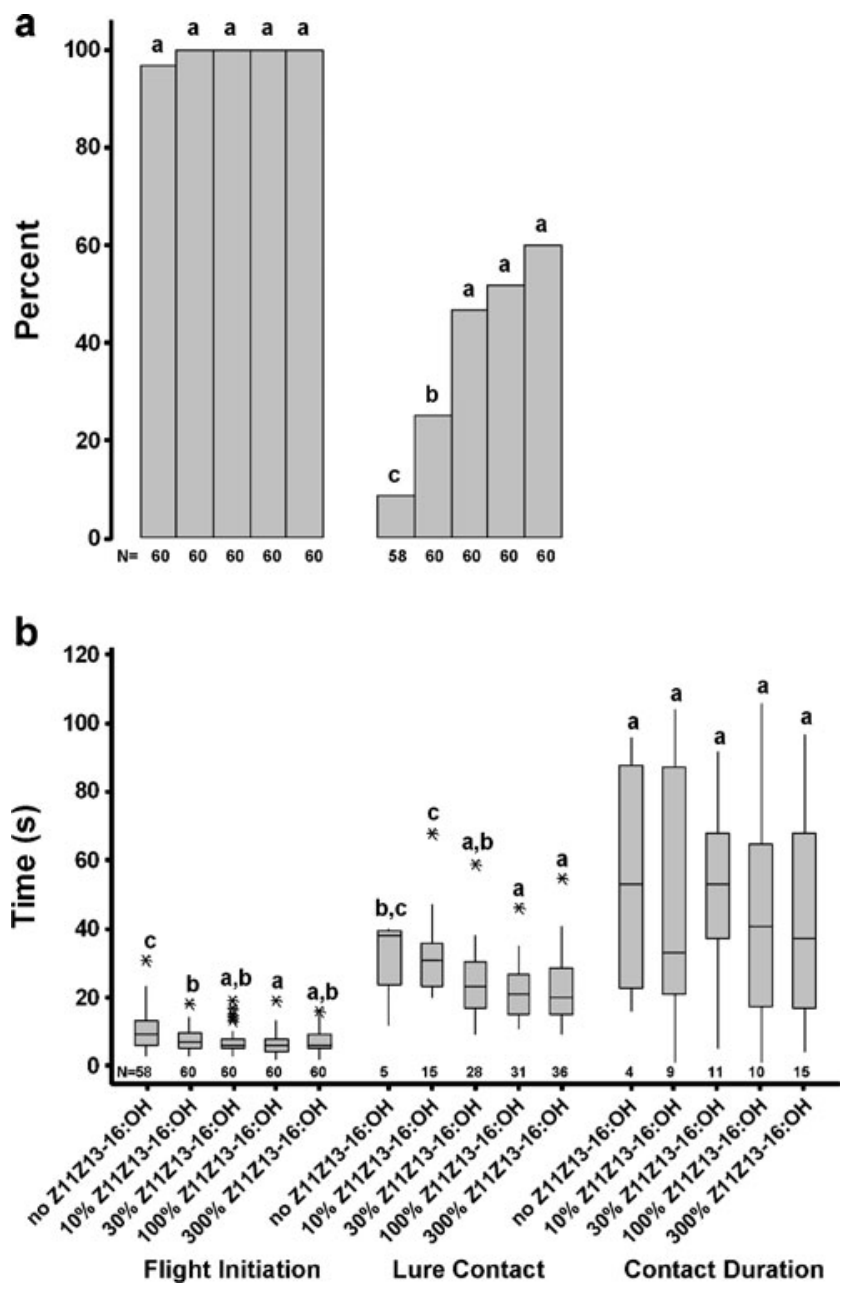

Fig. 3 Flight responses of male Amyelois transitella to pheromone blends with different amounts of $(11 Z, 13 Z)$-hexadecadien-1-ol (Z11Z13-16:OH). All treatments contain (11Z,13Z)-hexadecadienal $(10 \mathrm{ng}),(11 Z, 13 E)$-hexadecadien-1-ol and $(3 Z, 6 Z, 9 Z, 12 Z, 15 Z)$-tricosapentaene in a 100:5:15 ratio. The percentage of Z11Z13-16:OH is relative to the amount of $(11 Z, 13 Z)$-hexadecadienal. a Percentage of males that initiated flight within $60 \mathrm{sec}$ of exposure to lure, and percentage of males that contacted a lure. $\mathrm{N}$ indicates the number of males tested for each response. b Time to flight initiation (censored at $60 \mathrm{sec}$ ), time to lure contact, and contact duration (censored at $120 \mathrm{sec}$ ). Boxes indicate 25 th and 75 th percentiles, bars inside boxes are medians, whiskers denote the range, and asterisks represent outliers. $\mathrm{N}$ indicates the number of males exhibiting a response. Different letters above columns or boxes [in (a) or (b)] indicate significant $(P<0.05)$ differences blend, but all other metrics were not affected significantly by the presence or absence of this component.

A dose-response comparison using 1, 10, 100, and $1,000 \mathrm{ng}$, all with the 4-component blend at the optimized ratio, showed that the 10-ng dose elicited the best overall response when comparing across all metrics (Fig. 5). The 100 -ng dose resulted in a similar percentage of source contacts, but moths took longer to reach the source and

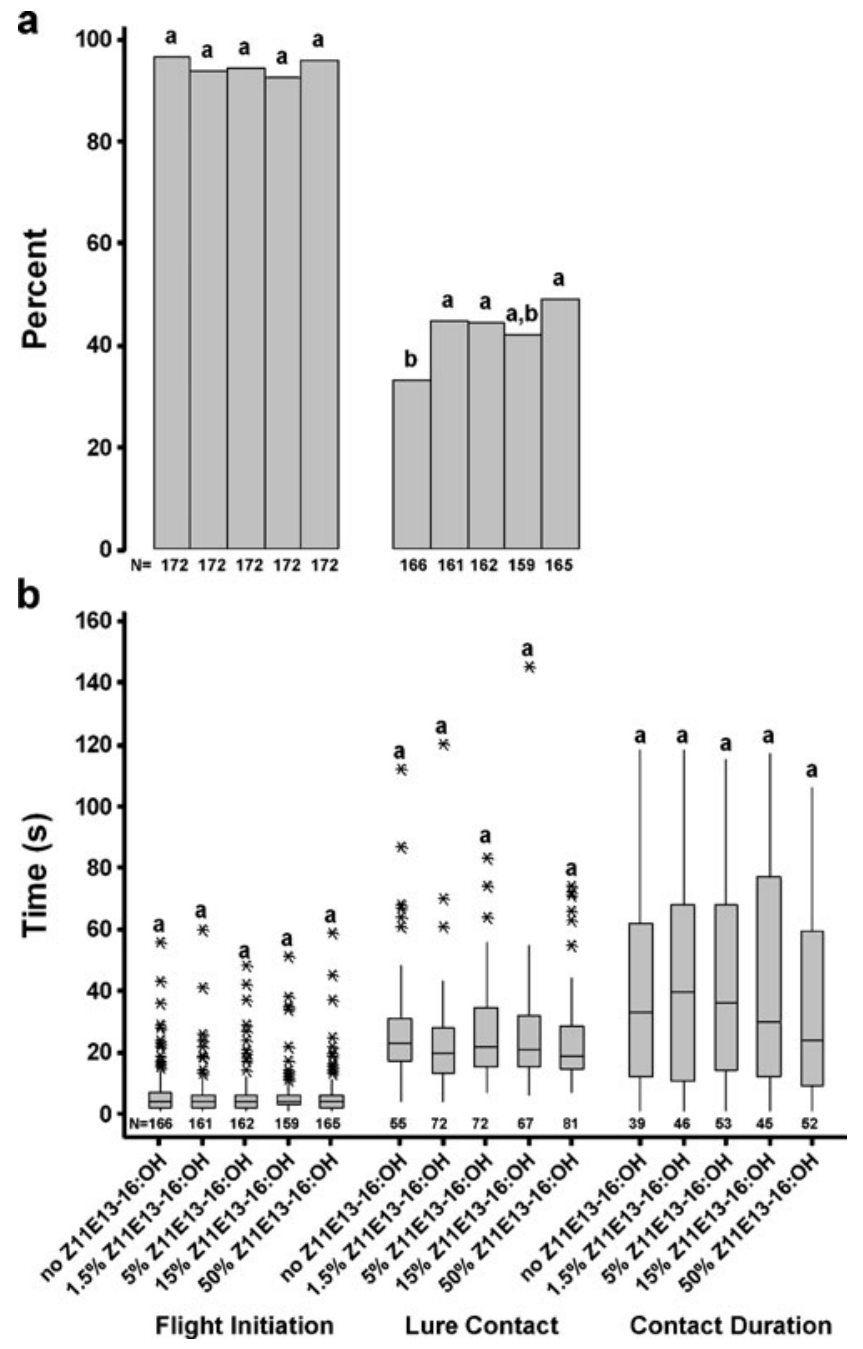

Fig. 4 Flight responses of male Amyelois transitella to pheromone blends with different amounts of $(11 Z, 13 E)$-hexadecadien-1-ol (Z11E13-16:OH). All treatments contain (11Z,13Z)-hexadecadienal (10 ng), (11Z,13Z)-hexadecadien-1-ol and (3Z,6Z,9Z,12Z,15Z)tricosapentaene in a 100:100:15 ratio. The percentage of Z11E13-16: $\mathrm{OH}$ is relative to the amount of $(11 Z, 13 Z)$-hexadecadienal. a Percentage of males that initiated flight within $60 \mathrm{sec}$ of exposure to lure, and percentage of males that contacted a lure. $\mathrm{N}$ indicates the number of males tested for each response. b Time to flight initiation (censored at $60 \mathrm{sec}$ ), time to lure contact, and contact duration (censored at $120 \mathrm{sec}$ ). Boxes indicate 25th and 75th percentiles, bars inside boxes are medians, whiskers denote the range, and asterisks represent outliers. $\mathrm{N}$ indicates the number of males exhibiting a response. Different letters above columns or boxes [in (a) or (b)] indicate significant $(P<0.05)$ differences 


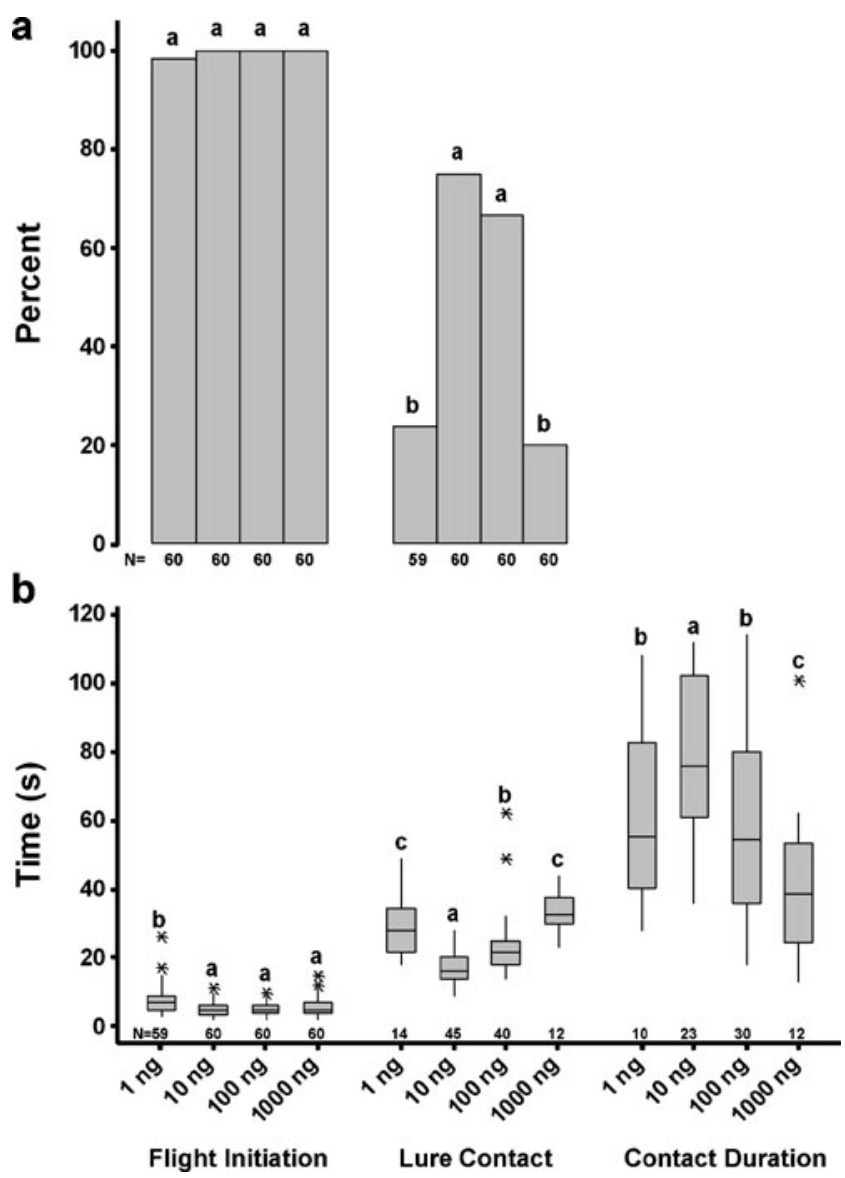

Fig. 5 Flight responses of male Amyelois transitella to 4 doses of a 100:100:5:15 ratio of the optimized 4-component pheromone blend of (11Z,13Z)-hexadecadienal, (11Z,13Z)-hexadecadien-1-ol, (11Z,13E)hexadecadien-1-ol, and $(3 Z, 6 Z, 9 Z, 12 Z, 15 Z)$-tricosapentaene. Dose $=$ amount of $(11 Z, 13 Z)$-hexadecadienal. a Percentage of males that initiated flight within $60 \mathrm{sec}$ of exposure to lure, and percentage of males that contacted a lure. $\mathrm{N}$ indicates the number of males tested for each response. b Time to flight initiation (censored at $60 \mathrm{sec}$ ), time to lure contact, and contact duration (censored at $120 \mathrm{sec}$ ). Boxes indicate 25 th and 75 th percentiles, bars inside boxes are medians, whiskers denote the range, and asterisks represent outliers. $\mathrm{N}$ indicates the number of males exhibiting a response. Different letters above columns or boxes [in (a) or (b)] indicate significant $(P<0.05)$ differences

remained in contact with the source for less time than for the $10 \mathrm{ng}$ dose. Both the 1-ng and the 1,000-ng doses resulted in markedly less source contact, longer latencies for source location, and shorter times in contact with the source, than were observed with the 10-ng dose.

The final experiment tested the effects of adding four other compounds that had been identified in pheromone gland extracts $[(3 Z, 6 Z, 9 Z, 12 Z, 15 Z)$-pentacosapentaene, ethyl hexadecanoate, Z11,Z13-16:COOEt, and Z11,Z13-16:Ac (Leal et al. 2005; Kuenen et al. 2010), to the optimized 4-component blend. None of these compounds, added to the 4-component blend in the proportions reported by Leal et al. (2005), significantly affected any measure of attraction (flight latency, proportion contacting the source, time to lure contact, or duration of lure contact) compared to the responses to the 4-component blend (data shown in supplementary material).

\section{Discussion}

The A. transitella pheromone was characterized initially as Z11,Z13-16:Ald by Coffelt et al. (1979a), but this component alone has proven to be minimally attractive in field tests over the last two decades (J.G.M. unpublished data). A number of possible additional components of the $A$. transitella pheromone were described by Leal et al. (2005), but this study did not define the subset of compounds (and their optimal ratio) that was both necessary and sufficient to obtain high attraction. Iterative refinement of the active blend did not prove trivial, requiring several years of wind tunnel and field trials (Kuenen et al. 2010). The present study has expanded on these previous reports by examining the roles of the various compounds in mediating the sequential steps in attraction to the source.

The wind-tunnel assay allowed assessment of the effects of a wide range of component combinations and ratios on sequential stages of the male moths' flight responses, from activation of quiescent moths, to initiation of flight, flight upwind along the pheromone plume, landing on the pheromone source, and time spent in contact with the odor source. As in past work with wind tunnel assays (e.g., Linn et al. 1988; Zhu et al. 1999; Trimble and Marshall 2008), the most diagnostic measure of attraction was the proportion of males reaching and contacting a point source of odor. However, in the present study, several other metrics, including latencies of response and lure contact, were consistent with the proportion of lure contact. The duration of lure contact was less discriminatory among treatments: once males landed on an odor source, they spent many seconds wing fanning and walking on the source, even if the blend was not as attractive as other blends.

The data from our study reinforced the work of Kuenen et al. (2010), by verifying that $C_{23}$ pentaene and Z11,Z1316:OH are components of the pheromone blend. Z11,E1316:OH had a less marked effect on the attraction of male moths to blends than $\mathrm{C}_{23}$ pentaene or Z11,Z13-16:OH, but still demonstrably affected the overall suite of behaviors.

One of the unexpected results from our studies was the general lack of sensitivity to component ratios by male A. transitella, especially given the importance of a precise component ratio in attraction of other moth species (reviewed in Cardé and Haynes 2004). This is likely because production of consistent blend ratios by females is more difficult when components have major structural 
differences (Roelofs and Cardé 1977). Furthermore, probably because $\mathrm{C}_{23}$ pentaene is biosynthesized in oenocytes and then transported to the pheromone gland (Wang et al. 2010), whereas the other fatty acid-derived components are biosynthesized de novo in the gland, it is possible that the blend emitted by females may change during a calling bout as the available $\mathrm{C}_{23}$ pentaene becomes depleted.

Male A. transitella were sensitive to the dose of pheromone, with attraction being maximal to $10 \mathrm{ng}$ and dropping to higher doses. This peak of response might be indicative of male response being linked to an optimum dose of pheromone, as has been noted with Grapholita molesta (Baker and Cardé 1979) and other moth species. Alternatively, the drop in attraction with increasing dose may be the result of small percentages of antagonistic impurities in the synthetic pheromone, which reached the threshold value required to affect the behavior of males, as the overall pheromone dose increased.

Our results, in combination with the data reported by Kuenen et al. (2010), suggest that other minor components found in gland extracts, including $(3 Z, 6 Z, 9 Z, 12 Z, 15 Z)$ pentacosapentaene, ethyl hexadecanoate, ethyl $(11 Z, 13 Z)$ hexadecadienoate, and $(11 Z, 13 Z)$-hexadecadienyl acetate (Leal et al. 2005; Kuenen et al. 2010), play no role in attraction of male $A$. transitella. However, the latter compound antagonizes attraction of a sympatric species, the meal moth Pyralis farinalis L. (Leal et al. 2005; Kuenen et al. 2010) that also uses both Z11,Z13-16:Ald and $C_{23}$ pentaene as components of its pheromone blend. Thus, this compound, and possibly some of the other minor components, may function as behavioral antagonists mediating interspecific interactions.

This characterization of attractive blends for A. transitella will help advance the development of field lures for surveillance and population monitoring of this important pest. However, the optimal blends described in this study are not yet ready for operational use, because there are lingering problems with longevity of lures in the field. In particular, Kuenen et al. (2010) reported that pheromone lures decreased dramatically in activity within a day of being deployed, for a variety of lure types that incorporated both an antioxidant and an ultraviolet light stabilizer in their formulations. This suggests that male $A$. transitella are extremely sensitive to degradation of one or more of the components of the blend under field conditions. We have not noted analogous decreases in activity of test solutions in our wind tunnel trials carried out under low-light conditions. This suggests that photooxidation processes occurring in sunlight may be responsible for the rapid loss of activity of field lures.

Past work on mating disruption of $A$. transitella has centered mainly on the use of widely spaced $\left(5\right.$ per ha $\left.^{-1}\right)$ "puffers", which dispensed metered doses of technical grade Z11,Z13-16:Ald in aerosol puffs at set intervals (Shorey and Gerber 1996). This disruptant system has been reported to be effective in suppressing male attraction to traps baited with virgin females and in reducing damage levels in almonds and pistachios (Higbee and Burks 2008), likely acting via the mechanism of "sensory impairment." Use of the complete blend might increase efficacy of disruption, however, especially if the pheromone were formulated with point sources that were attractive (Minks and Cardé 1988; Cardé and Minks 1995; Cardé 2007).

Acknowledgments We thank Tedros Berhane and Sima Raoufian for rearing the insects. Brad Higbee provided valuable insights into the behavior of the navel orangeworm. This project was supported by the Almond Board of California and the California Pistachio Commission.

Open Access This article is distributed under the terms of the Creative Commons Attribution Noncommercial License which permits any noncommercial use, distribution, and reproduction in any medium, provided the original author(s) and source are credited.

\section{References}

Ando, T., InOmate, S.-I., and Yamamoto, M. 2004. Lepidopteran sex pheromones, pp. 51-96, in S. Schulz (ed.). The Chemistry of Pheromones and Other Semiochemicals, Vol. 239, Topics in Current Chemistry. Springer, Berlin.

BAKER, T. C. and CARDÉ, R. T. 1979. Analysis of pheromonemediated behavior in male Grapholitha molesta, the oriental fruit moth (Lepidoptera: Tortricidae). Environ. Entomol. 8:956968.

CARDÉ, R. T. 2007. Using pheromones to disrupt mating of moth pests, pp. 122-169, in M. Kogan and P. Jepson (eds.). Perspectives in Ecological Theory and Integrated Pest Management. Cambridge University Press, Cambridge.

CARDÉ, R. T. and HAYNeS, K. F. 2004. Structure of the pheromone communication channel in moths, pp. 283-332, in R. T. Cardé and J. G. Millar (eds.). Advances in the Chemical Ecology of Insects. Cambridge University Press, Cambridge.

CARDÉ, R. T. and MinKS, A. K. 1995. Control of moth pests by mating disruption: successes and constraints. Annu. Rev. Entomol. 40:559-585.

Coffelt, J. A., Vick, K. W., Sonnet, P. E., and Doolittle, R. E. 1979a. Isolation, identification, and synthesis of a female sex pheromone of the navel orangeworm, Amyelois transitella (Lepidoptera: Pyralidae). J. Chem. Ecol. 5:955-966.

COFFelt, J. A., Vick, K. W., SOWER, L. L., and McClellan, W. T. 1979b. Sex pheromone mediated behavior of the navel orangeworm, Amyelois transitella. Environ. Entomol. 8:587-590.

HigBEE, B. S. and BURKS, C. S. 2008. Effects of mating disruption treatments on navel orangeworm (Lepidoptera: Pyralidae) sexual communication and damage in almonds and pistachios. J. Econ. Entomol. 101:1633-1642.

Justus, K. A., Murlis, J., Jones, C., and Cardé, R. T. 2002. Measurement of odor-plume structure in a wind tunnel using a photoionization detector and a tracer gas. Environ. Fluid Mech. $2: 115-142$

Kuenen, L. P. S., Rowe, H. C., Steffan, K., and Millar, J. G. 2001. Incomplete female sex pheromone of the navel orangeworm Amyelois transitella. California Pistachio Commission Annual Report Crop Year 2001-2002. p. 96. 
Kuenen, L. P. S., McElfresh, J. S., and Millar, J. G. 2010. Identification of critical secondary components of the sex pheromone of the navel orangeworm, (Lepidoptera: Pyralidae). J. Econ. Entomol. 103:314-330.

Leal, W. S., Parra-Pedrazzoli, A. L., Kaissling, K.-E., Morgan, T. I., Zalom, F. G., PesaK, D. J., Dundulis, E. A., BuRKs, C. S., and HigBeE, B. S. 2005. Unusual pheromone chemistry in the navel orangeworm: novel sex attractants and a behavioral antagonist. Naturwissenschaften 92:139-146.

LinN, C. E., JR., Hammond, A., Du, J., and Roelofs, W. L. 1988. Specificity of male response to multicomponent pheromones in noctuid moths Trichoplusia $n i$ and Pseudoplusia includens. J. Chem. Ecol. 14:47-57.

MinKS, A. K. and CARDÉ, R. T. 1988. Disruption of pheromone communication in moths: is the natural blend really most efficacious? Entomol. Exp. Appl. 49:25-36.

RoElofs, W. L. and CARDÉ, R. T. 1977. Responses of Lepidoptera to synthetic sex pheromone chemicals and their analogues. Annu. Rev. Entomol. 22:377-405.
SHOREY, H. H. and GERBER, R. G. 1996. Use of puffers for disruption of sex pheromone communication among navel orangeworm moths (Lepidoptera: Pyralidae) in almonds, pistachios, and walnuts. Environ. Entomol. 25:1154-1157.

TrimBle, R. M. and MARShall, D. B. 2008. Relative attractiveness of incomplete and complete blends of synthetic pheromone to male obliquebanded leafroller (Lepidoptera: Tortricidae) moths in a flight tunnel and in apple orchards: Implications for sexpheromone mediated mating disruption. Environ. Entomol. 37:366-373.

Wang, H.-L., ZhaO, C.-H., Millar, J. G., Cardé, R. T., and LÖFSTEDT, C. 2010. Biosynthesis of unusual moth pheromone components involves two distinctly different pathways in the navel orangeworm, Amyelois transitella. J. Chem. Ecol. 36:535-547.

Zhu, J., Ryne, C., Unelius, C. R., VAleur, P. G., and LöFstedt, C. 1999. Reidentification of the female sex pheromone of the Indian meal moth, Plodia interpunctella: evidence for a fourcomponent pheromone blend. Entomol. Exp. Appl. 92:137146. 